\title{
EVALUATION OF THE FREQUENCY OF AUTOIMMUNE CUTANEOUS DISORDERS IN PATIENTS WITH CELIAC DISEASE.
}

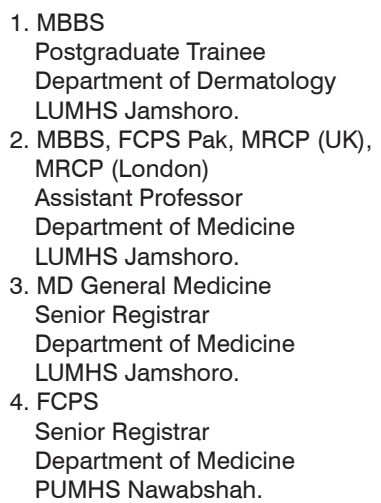

Correspondence Address: Dr. Muhammad Aslam Rind Banglo No.A12, Sharif Excuare Hussainabad,

Hyderabad.

draslamrind82@hotmail.com

Article received on: 04/02/2019

Accepted for publication: 25/06/2019

\begin{abstract}
Quratulain 1 , Muhammad Aslam Rind ${ }^{2}$, Maria Nazir ${ }^{3}$, Muhammad Saleem Rind ${ }^{4}$
ABSTRACT... Objectives: To evaluate the frequency of autoimmune cutaneous disorders in patients with celiac disease. Study Design: This descriptive cross sectional study. Setting: conducted at Dermatological Department of Liaquat University Hospital Hyderabad/ Jamshoro. Period: From $1^{\text {st }}$ March 2015 to $31^{\text {st }}$ August 2015. Material \& Methods: Total 352 patients of age 15 to 45 years \& either gender having celiac disease were enrolled consecutively. Patients with history of abnormal serological test results and already on gluten free diet, known cases of diabetes mellitus, tuberculosis, systemic lupus erythematosus (SLE), secondary syphilis, hyper or hypothyroidism, iron deficiency anemia, pernicious anemia, Addison disease and pituitary insufficiency were excluded. The data was analyzed using SPSS version 16. The frequency/ percentage were calculated for autoimmune cutaneous disorders i-e; Dermatitis herpetiformis, Alopecia areata, vitiligo \& psoriasis \& for gender. The mean and standard deviation (SD) were calculated for age, duration of celiac disease and duration of cutaneous disorders. Stratified analysis was done followed by applying chi-square with a $p$ value $<0.05$ taken as significant. Results: The mean age was $29.83 \pm 8.91$ years (Range 15-45). The mean duration of celiac disease was $5.99 \pm 2.30$ years (Range: $1-10$ years). The Mean duration of cutaneous manifestation was $7.55 \pm 3.30$ months (Range: 3 to 15). Slight less than half of all patients were females $46.3 \%$. More than two third patients i-e; $69 \%(n=243)$ in this study were from rural areas. About $10.2 \%$ patients had dermatitis herpatiformis, $6.3 \%$ patients had alopecia areata, $2.6 \%$ patients had Vitiligo while $16.5 \%$ had psoriasis. The gender, age of patients, duration of $C D \&$ duration of development of skin symptoms were non-significant effect modifiers for the frequency of cutaneous manifestations among CD patients. Conclusions: Therefore, it concludes that psoriasis, dermatitis herpatiformis \& alopecia areata are quite prevalent in CD patients. Vitiligo is rare condition. Screening for such autoimmune cutaneous manifestations and proper treatment of all such patients should be carried out routinely.
\end{abstract}

Key words: Alopecia Areata, Celiac Disease, Dermatitis Herpatiformis, Enteropathy, Gluten, Psoriasis Vitiligo.

Article Citation: Quratulain, Rind MA, Nazir M, Rind MS. Evaluation of the frequency of Autoimmune Cutaneous Disorders in patients with Celiac disease. Professional Med J 2019; 26(12):2105-2111.

DOI: 10.29309/TPMJ/2019.26.12.3216

\section{INTRODUCTION}

Celiac disease (CD) is characterized by malabsorption of nutrients, chronic inflammation and damage of the small intestinal mucosa caused by the ingestion of gliadin fraction of wheat gluten and similar alcohol-soluble proteins of barley and rye in genetically susceptible subjects. ${ }^{1}$ Celiac disease $(C D)$ is an immune-mediated disease which is triggered by gluten exposurein genetically sensitive individuals. ${ }^{1}$ It affects both children and adults and can present at any age. Celiac disease affects approximately $1 \%$ of the population, and is one the most common lifelong condition in the
United States. ${ }^{2}$ The clinical presentation of CD is extremely heterogeneous. Typical symptoms include chronic diarrhea, abdominal distension, and failure to thrive..$^{2,3}$ However, only a few patients with $C D$ show clinical malabsorption while most patients have subtle symptoms. ${ }^{1}$ The aim of this study is to report commoner CD associated skin manifestations. The prevalence of CD has changed over the last 30-40 years with the help of highly sensitive serologic tests, such as anti-endomysial antibodies (EMA) and antitissue trans-glutaminase (tTG) antibodies, which have made CD diagnosis easier in subclinical 
cases and several risk groups. ${ }^{1,3,4}$ Screening studies show a high prevalence of CD (between 1/130-1/300) among both healthy children and adult populations in European countries. The prevalence of celiac disease in Pakistan is unknown, however it is felt to be a very common disorder both in children and adults. ${ }^{3}$ Earlier research suggests that $C D$ is associated with number of autoimmune diseases. ${ }^{1}$ Dermatitis herpetiformis $(\mathrm{DH})$ is a skin manifestation of $\mathrm{CD}$ presenting with blistering rash and pathognomonic cutaneous IgA deposits. ${ }^{4}$ Dermatitis herpetiformis is most common in Caucasians of northern European descent with a prevalence of 11.2 and incidence of $0.98 \%$ per $1,00,000$ people. $^{5}$ Among the inflammatory skin diseases improved by gluten free diet, psoriasis is one of the most important, prevalence of psoriasis reported by Birkenfeld et al, Montesu et al was $29 \%$ and $2 \% .^{6,7}$ Alopecia areata (AA) is an autoimmune disease with a frequency ranging from $0.7 \%$ to $3.8 \% 8$ and vitiligo occurs with a frequency of $0.1-2 \%$ in various population. ${ }^{8,9}$ Celiac disease is, however, still under recognized as a cause of various skin manifestations. ${ }^{10,11}$ Therefore, it is important to recognize these patients due to significant prognostic implications. This study was conducted for the evaluation of frequency of autoimmune cutaneous disorders and to evaluate their prevalence in patients with celiac disease. The study also provided the highlighting the importance of a close collaboration between gastroenterologists and dermatologists and knowledge to health care workers as for as management strategy is concerned.

\section{OBJECTIVE}

To evaluate the frequency of autoimmune cutaneous disorders in patients with celiac disease.

\section{OPERATIONAL DEFINITIONS}

\section{Celiac Disease}

It was considered on the basis of following parameters:

Abnormal serological test result: presence of both was

labeled as celiac disease:
- IgA endomysial antibody

- IgA tissue transglutaminase antibody

\section{AUTOIMMUNCE CUTANEOUS DISORDERS}

\section{a. Dermatitis Herpatiformus}

Was regarded as a cutaneous variant of celiac disease. It is a characteristic skin rash consisting of pruritic papulo-vesicles over extensor surfaces of the extremities and over the trunk, scalp, and neck.

\section{b. Alopecia Areata}

Alopecia areata (AA) is an autoimmune disease that presents as non-scarring hair loss. It was diagnosed on the following parameters:

\section{Clinical Examination}

\begin{tabular}{|l|l|l|}
\hline \multicolumn{1}{|c|}{ Bald Patches Scalp $\begin{array}{c}\text { Beard } \\
\text { Area }\end{array}$} & Eyebrows \\
\hline Number of bald patches & & \\
\hline Size of bald patches & & \\
\hline
\end{tabular}

Biopsy: histologically shows peri-follicular lymphocyte infiltration.

c. VITILIGO: The diagnosis of vitiligo is based upon the clinical presence of depigmented patches of skin. (Examination with a Wood's lamp is useful for highlighting areas of pigment loss on lightly-pigmented patients).

d. PSORIASIS: Psoriasis is a common chronic skin disorder typically characterized by erythematous papules and plaques with a examination; there were no special blood tests or diagnostic procedures. silver scale, the diagnosis of psoriasis can be made by history and physical

\section{MATERIAL AND METHODS}

\section{Study Design}

Descriptive (Cross-sectional study).

\section{Setting}

Dermatological department of Liaquat University Hospital Hyderabad/ Jamshoro. Some sample 
was collected from General Medicine department as well.

\section{Study Duration}

Six months

\section{Sample Size}

Prevalence of dermatitis herpatiformis $11.2 \%$, psoriasis 2\%-29\%, alopecia areata $3.8 \%$ and vitiligo $0.1-2 \%$. By taking prevalence of vitilgo $2 \%$, $\mathrm{d}=1.5 \%, \mathrm{n}=352$ patients with celiac disease.

\section{Sample Technique}

Non probability consecutive sampling

\section{SAMPLE SELECTION}

\section{Inclusion Criteria}

1. Diagnosed cases of celiac disease (abnormal serological test results) presenting at Liaquat University Hospital.

2. Patients age 15 to 45 years of age.

3. Either Gender.

\section{Exclusion Criteria}

1. Patients who had abnormal serological test results and already on gluten free diet.

2. The known cases of diabetes mellitus, tuberculosis, systemiclupus erythematosus (SLE), secondary syphilis, hyper or hypothyroidism, iron deficiency anemia, pernicious anemia, Addison disease and pituitary insufficiency (on the basis of history, clinical examination and lab investigation) and the non-cooperative patients or who refused to give consent or not interested to participate in the study was considered as exclusion criteria.

\section{DATA COLLECTION PROCEDURE}

This study was conducted on the patients who meet the inclusion criteria of the study. All the diagnosed cases (operational definition) of celiac disease admitted in Dermatological ward and OPD was enrolled and entered in the study. For serology $5 \mathrm{ml}$ venous blood sample was collected for IgAendomysial antibody and IgA tissue transglutaminase antibody. Though gold standard test for diagnosing celiac disease is endoscopy guided jejunal biopsy, but our study totally relied on antibodies because of non- availability of biopsy in our setup. The primary outcome variable was the evaluation of frequency of autoimmune cutaneous disorders and their prevalence in patients with celiac disease. Informed consent was taken from all patients or their parents.

DATA ANALYSIS: The data of all patients were entered and analyzed in SPSS version 16. The frequency and percentage (\%) were calculated for autoimmune cutaneous disorders (as mentioned above) i-e; DHA, AA, vitiligo \& psoriasis in patients with celiac disease. The frequency and percentage (\%) were also be calculated for gender. The mean and standard deviation (SD) were calculated for age, duration of celiac disease and duration of cutaneous disorders. The stratification was done for age, gender \& duration of disease \& cutaneous disorders to see the effect on outcome and to control the confounders. The post stratification chi-square test was applied at 95\% confidence interval and the $p$-value $\leq 0.05$ was considered as statistically significant.

\section{RESULTS}

Total 352 patients having celiac disease were taken as sample for this study to evaluate the frequency of autoimmune cutaneous manifestations in them. The mean age was $29.83 \pm 8.91$ years with range of 16-45 years. The mean duration of celiac disease was $5.99 \pm 2.30$ years (Range: $1-10$ years). (Table-I).

\begin{tabular}{|l|c|c|c|c|}
\hline \multicolumn{1}{|c|}{$\mathbf{n = 3 5 2}$} & Mean & $\begin{array}{c}\text { Standard } \\
\text { Deviation }\end{array}$ & Minimum & Minimum \\
\hline Age (Years & 29.83 & 8.91 & 16 & 45 \\
\hline $\begin{array}{l}\text { Duration of } \\
\text { Disease (Years) }\end{array}$ & 5.99 & 2.30 & 1 & 10 \\
\hline $\begin{array}{l}\text { Duration of } \\
\text { Cutaneous } \\
\text { Manifestation } \\
\text { (Months) }\end{array}$ & 7.55 & 3.30 & 3 & 15 \\
\hline
\end{tabular}

Table-I. Descriptive statistics on quantitative variable.

Mean duration of cutaneous manifestation was noted to be $7.55 \pm 3.30$ months which ranged from 3 to 15 months. (Table-I). Slight less than half of all patients were females $46.3 \%(n=163)$ and the males were $53.7 \%(n=189)$. 
It was noted in this study that $43.2 \%(n=152)$ patients were young and were of age between $15-25$ years. Further about $22.2 \%(n=78)$ were between the age of 26 to 35 years and a third i-e; $34.7 \%(n=122)$ patients were of age categories 36 to 45 years. (Figure-1).

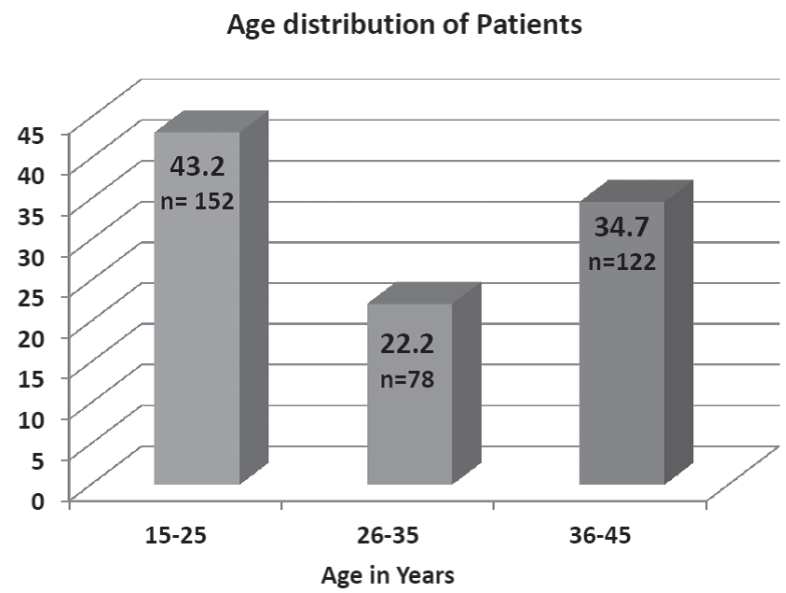

Figure-1. Age categories of patients.

More than two third patients i-e; $69 \%(n=243)$ in this study were from rural areas while those from urban areas were $31 \%(n=109)$. Regarding duration of $C D$ it was found that 4 percent patients had disease since previous less than two years. Those having disease duration of 3-4 years were $29 \%$, 5-6 years duration were $22.4 \%$, 7-8 years duration were $27.3 \%$ while $17.3 \%$ were patients who had CD since 9-10 years. (Table-II).

\begin{tabular}{|l|c|c|}
\hline $\begin{array}{c}\text { Disease duration } \\
\text { (Years) }\end{array}$ & Frequency & Percent \\
\hline Up to 2 & 14 & 4.0 \\
\hline $3-4$ & 102 & 29.0 \\
\hline $5-6$ & 79 & 22.4 \\
\hline $7-8$ & 96 & 27.3 \\
\hline $9-10$ & 61 & 17.3 \\
\hline Total & 352 & 100.0 \\
\hline
\end{tabular}

Table-II. Disease duration (in years) among patients of celiac disease.

Patients presenting with cutaneous manifestation of less than 4 months were $21.6 \%$; duration of 5-8 months were $38.6 \%$, duration of 9-12 months were $31.3 \%$ while $8.5 \%$ were patients who had cutaneous manifestation since 13 or more than that
months.Regarding the frequency of autoimmune cutaneous manifestations among celiac disease patients it was found that $10.2 \%(n=36)$ patients had dermatitis herpatiformis $(\mathrm{DH}), 6.3 \%(n=22)$ patients had alopecia areata (AA), 2.6\% $(n=9)$ patients had Vitiligo while 16.5\% $(n=58)$ patients had psoriasis. (Figure-2).

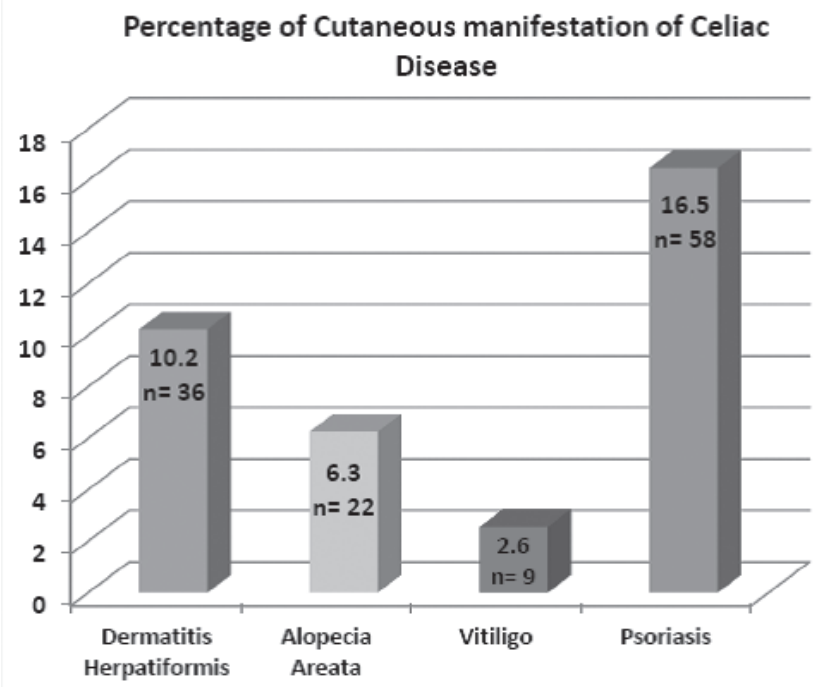

Figure-2. Frequency of cutaneous manifestation among patients of celiac diseases.

In this study stratification with respect to gender, age, duration of CD and duration of cutaneous manifestations was done which revealed that the gender was a non-significant effect modifier for the frequency of cutaneous manifestations among CD patients. Accordingly; leaving only $\mathrm{DH}$, other cutaneous manifestations like AA, Vitiligo \& psoriasis were more common in female gender. ( $P$ values $=0.223,0.443,0.409 \& 0.318)$. Further the stratified analysis revealed that age of patients was a nonsignificant effect modifier with which the frequencies of cutaneous manifestations in celiac disease patients increased with increasing age leaving only vitiligo. ( $P$ values $=0.259,0.248$, $0.349 \& 0.524)$. The duration of disease was an effect modifier for the frequency of cutaneous manifestations among CD patients. Accordingly; $\mathrm{DH} \&$ psoriasis decreased while other cutaneous manifestations like AA \& Vitiligo increased with increasing disease duration. All these findings were not significant. $(\mathrm{P}$ values $=0.321,0.140$, 0.643 \& 0.123; Table-III). 


\begin{tabular}{|c|c|c|c|c|}
\hline \multirow{2}{*}{$\begin{array}{c}\text { Disease Duration in } \\
\text { Years }\end{array}$} & \multicolumn{4}{|c|}{ Frequency of Cutaneous Manifestation } \\
\hline & DH & AA & Vitiligo & Psoriasis \\
\hline \multirow{2}{*}{ Up to 2} & 4 & 0 & 0 & 6 \\
\hline & $28.6 \%$ & $0.0 \%$ & $0.0 \%$ & $42.9 \%$ \\
\hline \multirow{2}{*}{$3-4$} & 9 & 11 & 3 & 19 \\
\hline & $8.8 \%$ & $10.8 \%$ & $2.9 \%$ & $8.6 \%$ \\
\hline \multirow{2}{*}{$5-6$} & 6 & 5 & 1 & 10 \\
\hline & $7.6 \%$ & $6.3 \%$ & $1.3 \%$ & $12.7 \%$ \\
\hline \multirow{2}{*}{$7-8$} & 11 & 3 & 4 & 13 \\
\hline & $11.15 \%$ & $3.1 \%$ & $4.2 \%$ & $13.5 \%$ \\
\hline \multirow{2}{*}{$9-10$} & 6 & 3 & 1 & 10 \\
\hline & $9.8 \%$ & $4.9 \%$ & $1.6 \%$ & $16.4 \%$ \\
\hline Total & 36 & 22 & 9 & 58 \\
\hline \multirow{2}{*}{ P-Value } & $10.2 \%$ & $6.2 \%$ & $2.6 \%$ & $16.5 \%$ \\
\hline & 0.321 & 0.140 & 0.643 & 0.123 \\
\hline
\end{tabular}

Table-III. Effect of diseases duration on frequency of cutaneous manifestation in patients of celiac disease.

Finally; the symptoms (cutaneous manifestations) also showed varied picture with duration of development. Accordingly; it was noted that leaving only Vitiligo, the frequency of other cutaneous manifestations like $\mathrm{AA}, \mathrm{DH}$ \& psoriasis increased with increasing duration of symptoms. $(P$ values $=0.223,0.504,0.257 \& 0.154)$.

\section{DISCUSSION}

Celiac disease (CD), an immune-mediated enteropathy which is caused by the ingestion of gluten in susceptible individuals is the most common genetic disease of mankind overall affecting $>3$ million people worldwide..$^{12,13,14}$ Most people with celiac disease or gluten sensitivity are never diagnosed, and are instead misdiagnosed as having chronic fatigue syndrome, fibromyalgia, irritable bowel syndrome and other conditions. Yet; the world prevalence of $C D$ is estimated at 1:266 people. ${ }^{10,12}$ The major problem in diagnosing celiac disease is the multifaceted clinical picture of the condition. CD presenting with a severe malabsorption syndrome is an exception now a days. Instead, the disease seems to express itself with milder and atypical symptoms of which sometimes the presenting symptoms are cutaneous manifestations. The best known extra intestinal manifestation of celiac disease is dermatitis herpetiformis which is a cutaneous manifestation. ${ }^{15,16} \mathrm{CD}$ is not an uncommon diagnosis in Pakistan. The current study was conducted to evaluate the frequency of autoimmune cutaneous manifestations of CD at
Hyderabad which is a major resort point for such type of patients coming from peripheral areas of Sindh (>two third patients i-e; $69 \%$ in this study were from rural Sindh). Out of sample of 352 in this study; males were in majority i-e; $53.7 \%$ the current study noted that mean \pm SD age of patients was $29.83 \pm 8.91$ years. It was also noted that majority of patients were of younger age. Other studies have also documented that patients of CD which present with skin lesions usually are of younger age. Simultaneously; it was noted in this study that mean \pm SD duration of celiac disease was $5.99 \pm 2.30$ years while; mean \pm SD duration of cutaneous manifestation was noted to be $7.55 \pm 3.30$ months which ranged from 3 to 15 months. It is important to note that duration of skin lesions ranged from 3 to 15 months in which case it is reasonable to assume that due to lack of awareness, misdiagnosis, financial unaffordability (prevalent in rural Sindh), people come lately to tertiary care centers for the skin lesions. Psoriasis was most common autoimmune cutaneous manifestations in CD patients in this study. It was detected in $16.5 \%$ patients followed by dermatitis herpatiformis among $10.2 \%$ \& alopecia areata present in $6.3 \%$. Vitiligo was less common and only $2.6 \%$ CD patients were affected with it. Other studies have reported more or less mimicking results. In some studies one condition (e.g; dermatitis herpatiformis) was slightly more prevalent while other studies reported other condition (Psoriasis) being commoner. ${ }^{6,9,12}$ Overall no any much difference was detected 
between multiple studies. Abenavoli L, et al have reported that the incidence of dermatitis herpetiformis is highest in Ireland and Sweden and rare in Asia. Other study by Salmi TT, et al., ${ }^{4}$ reported that dermatitis herpetiformis is most common cutaneous manifestations among CD patients in Finland. On the other hand, different authors evaluated the prevalence of $C D$ in patients affected by psoriasis, showing a high frequency of CD (4.34\%) in psoriatic patients. It had been documented by some of studies that those patients who have celiac disease with dermatitis herpetiformis are at greater risk than the general population for developing one or more of the associated autoimmune diseases like Insulin Dependent Diabetes Mellitus (Type 1 Diabetes), Thyroid diseases, Addison's disease, Autoimmune chronic active hepatitis, Sjogren's syndrome, Systemic lupus erythematosus \& Myasthenia gravis etc. ${ }^{13,14,17,18}$ Leaving a few, most of studies have not evaluated the effect of age, gender and duration of disease over the frequency of cutaneous manifestations among CD patients. We; in current study designed to assess this effect. Accordingly; we noted that the gender, age of patients, duration of CD \& duration of development of skin symptoms were non-significant effect modifiers for the frequency of cutaneous manifestations among CD patients. Further it was noted that alopecia areata, vitiligo \& psoriasis were more common in female gender while the frequencies of almost all skin lesions increased with increasing age while it was important to note that larger the duration of $C D$, higher was the frequency of alopecia areata \& Vitiligo. Variability in prevalence and effect modification in this study as compared to other studies is thought to be due to difference of area, duration of disease, skin exposure to light, study design and sample size. Partial or complete treatment of sing lesions might also have played some role in the difference of results (between the studies) which was not collected by the current study. The current study has put light on an important but ignored aspect of celiac disease and accounted for the morbidity from which CD patients suffer. We recommend that all such patients should be screened for these autoimmune cutaneous manifestations and treated properly in order to prevent cutaneous complications and undue morbidity which our CD patients face. It should be followed by strong vigilance of development of these skin lesions. This way extra burden on health care system may also be shaded off.

Copyright $@ 25$ June, 2019.

\section{REFERENCES}

1. Neuhausen SL, Steele L, Ryan S. Co-occurrence of celiac disease and other autoimmune diseases in celiacs and their first degree relatives. J Autoimmun. 2008; 31:160-5.

2. Holmes G, Catassi C, Fasano A. Fast facts: Celiac disease 2nd ed. Abingdon: Health Press. 2009.

3. Rashid M, Khan AG. Celiac disease in Pakistan: Challenges and opportunities. J Ayub Med Coll Abbottabad. 2009; 21 (3).

4. Salmi TT, Hervonen $\mathrm{K}$, Kautiainen $\mathrm{H}$, Collin incidence of dermatitis herpetiformis: A Finland. Brit J Dermatol. $2011 ; 165: 354-9$.

5. Bangert CA, Costner MI. Methotrexate in dermatology. Dermatol Ther. 2007; 20:216- 28.

6. Birkenfeld S, Dreiher J, Weitzman D, Cohen AD. Coeliac disease associated with psoriasis. Brit $\mathrm{J}$ Dermatol. 2009; 161:1331-34.

7. Montesu MA, Dessi-Fulgheri C, Pattaro C, Ventura V, Satta R, Cottoni F. Association between psoriasis and coeliac disease?: A case-control study. Acta Dermato-Venereologica. 2011; 91:92-3.

8. Tan E, Tay YK, Goh CL, Giam YC. The pattern and profile of alopecia areata in Singapore: A study of 219 Asians. International journal of dermatology. 2002; 41(11):748-53.

9. Alkhateeb A, Fain PR, Thody A, Bennett DC, Spritz RA. Epidemiology of vitiligo and associated autoimmune diseases in Caucasian probands and their families. Pigment Cell Res. 2003; 16(3):208-14.

10. Caproni M, Bonciolini V, D‘Errico A, Antiga E, Fabbri $P$. Celiac disease and dermatologic manifestations: Many skin clue to unfold gluten-sensitivity enteropathy. Gastroenterol Res Pract. 2012; 2012:952753.

11. Lewin KJ, Riddell RH, Weinstein WM. Small bowel mucosal disease. In: Gastrointestinal pathology and its clinical applications Vol-Il. Tokyo: Igaku- Shoin. 1992: 750-811. 
12. Cooke WT, Holmes GKT. Definition and epidemiology. In: Coeliac disease. NewYork: Churchill Livingtone. 1984: 11-22.

13. Ivarsson A. The Swedish epidemic of coeliac disease explored using an epidemiological approach: Some lessons to be learnt. Best Pract Res Clin Gastroenterol. 2005; 19:425-40.

14. Fasano A, Catassi C. Current approaches to diagnosis and treatment of celiac disease: An evolving spectrum. Gastroenterology. 2001; 120:636-51.

15. Fasano A, Berti I, Gerarduzzi T, Not T, Colletti RB, Drago $S$, et al. Prevalence of celiac disease in at risk and not-at-risk groups in the United States: A large multicenter study. Arch Intern Med. 2003; 163:286-92.
16. Ivarsson A, Hernell O, Stenlund H, Persson LA. Breastfeeding protects against celiac disease. Am J Clin Nutr. 2002; 75:914-21.

17. Memeo L, Jhang J, Hibshoosh H, Green PH, Rotterdam $H$, Bhagat $G$. Duodenal intraepithelial lymphocytosis with normal villous architecture: Common occurrence in $\mathbf{H}$ pylori gastritis. Mod Pathol. 2005; 18:1134-44.

18. Arentz-Hansen $\mathrm{H}$, Fleckenstein $\mathrm{B}$, Molberg $\mathrm{O}$, Scott $\mathrm{H}$, Koning $F$, Jung $G$, et al. The molecular basis for oat intolerance in patients with celiac disease. PLoS Med. 2004; 1(1):e1.

\begin{tabular}{|c|c|c|}
\hline \multicolumn{3}{|c}{ AUTHORSHIP AND CONTRIBUTION DECLARATION } \\
\hline Sr. \# & Author(s) Full Name & \multicolumn{1}{|c|}{ Contribution to the paper } \\
\hline 1 & Quratulain & $\begin{array}{l}\text { Protocol writing, Study design } \\
\text { and concept, Data collection, } \\
\text { Analysis, Manuscript writing. } \\
\text { Protocol writing, Study design } \\
\text { and concept, Data collection, } \\
\text { Analysis, Manuscript writing. } \\
\text { Datacollection, Analysis, } \\
\text { Protocol writing, Manuscript } \\
\text { review. } \\
\text { Data analysis, Data collection, } \\
\text { Study design, manuscript } \\
\text { writing and review. }\end{array}$ \\
\hline
\end{tabular}

